\title{
Multi-cycle reconfigurable THz extraordinary optical transmission using chalcogenide metamaterials
}

\author{
Tun Cao $\oplus^{1+*}$, Meng $\operatorname{Lian}^{1 \dagger}$, Xieyu Chen ${ }^{2 \dagger}$, Libang Mao ${ }^{1 \dagger}$, Kuan Liu ${ }^{1}$, \\ Jingyuan Jia ${ }^{1}$, Ying $\mathrm{Su}^{1}$, Haonan $\mathrm{Ren}^{1}$, Shoujun Zhang ${ }^{2}$, Yihan $\mathrm{Xu}^{2}$, \\ Jiajia Chen ${ }^{2}$, Zhen Tian ${ }^{2 *}$ and Dongming Guo ${ }^{3 *}$
}

\begin{abstract}
Metamaterials composed of metallic antennae arrays are used as they possess extraordinary optical transmission (EOT) in the terahertz $(\mathrm{THz})$ region, whereby a giant forward light propagation can be created using constructive interference of tunneling surface plasmonic waves. However, numerous applications of $\mathrm{THz}$ meta-devices demand an active manipulation of the THz beam in free space. Although some studies have been carried out to control the EOT for the THz region, few of these are based upon electrical modulation of the EOT phenomenon, and novel strategies are required for actively and dynamically reconfigurable EOT meta-devices. In this work, we experimentally present that the EOT resonance can be coupled to optically reconfigurable chalcogenide metamaterials which offers a reversible all-optical control of the $\mathrm{THz}$ light. A modulation efficiency of $88 \%$ in transmission at $0.85 \mathrm{THz}$ is experimentally observed using the EOT metamaterials, which is composed of a gold ( $\mathrm{Au}$ ) circular aperture array sitting on a non-volatile chalcogenide phase change material $\left(\mathrm{Ge}_{2} \mathrm{Sb}_{2} \mathrm{Te}_{5}\right)$ film. This comes up with a robust and ultrafast reconfigurable EOT over 20 times of switching, excited by a nanosecond pulsed laser. The measured data have a good agreement with finite-element-method numerical simulation. This work promises THz modulators with significant on/off ratios and fast speeds.
\end{abstract}

Keywords: metamaterials; extraordinary optical transmission; surface plasmon resonance; reconfigurable; phase change materials

Cao T, Lian M, Chen XY, Mao LB, Liu K et al. Multi-cycle reconfigurable THz extraordinary optical transmission using chalcogenide metamaterials. Opto-Electron Sci 1, 210010 (2022).

\section{Introduction}

Terahertz (THz) electromagnetic (EM) waves residing in the regime between infrared and microwave spectra have attracted intense attention due to their appealing advantages of excellent transparency, low photon energy, and high spectral resolution. Many works have exploited the interferences of $\mathrm{THz}$ waves with matter, for which promising applications include security inspection, non-destructive evaluation, medical imaging, chemical identification, and high-speed wireless communications ${ }^{1-4}$. Yet, a complete exploration of constructive applications over the $\mathrm{THz}$ regime is limited owing to the unavailability of

${ }^{1}$ School of Optoelectronic Engineering and Instrumentation Science, Dalian University of Technology, Dalian 116024, China; ${ }^{2}$ Center for Terahertz Waves and College of Precision Instrument and Optoelectronics Engineering, Tianjin University, Tianjin 300072, China; ${ }^{3}$ School of Mechanical Engineering, Dalian University of Technology, Dalian 116024, China.

†These authors contributed equally to this work.

*Correspondence: T Cao, E-mail: caotun1806@dlut.edu.cn; Z Tian, E-mail: tianzhen@tju.edu.cn; DM Guo, E-mail: guodm@dlut.edu.cn Received: 14 October 2021; Accepted: 17 November 2021; Published online: 30 November 2021

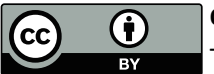

Open Access This article is licensed under a Creative Commons Attribution 4.0 International License.

To view a copy of this license, visit http://creativecommons.org/licenses/by/4.0/.

(C) The Author(s) 2022. Published by Institute of Optics and Electronics, Chinese Academy of Sciences. 
appropriate responses in the $\mathrm{THz}$ region for numerous naturally present materials $s^{5}$. Metamaterials are artificially structured materials whose EM properties are mainly decided by the meta-atom profile, enabling them to produce novel EM functions that are not present in natural materials, such as cloaking, negative refractive index, and superlens ${ }^{6-11}$. Moreover, the light-matter interference can be considerably enhanced since the metaatom offers intense confinement of EM-fields in a subwavelength area over almost the whole EM-spectrum. Such metamaterials can be used to develop various novel $\mathrm{THz}$ devices, which are traditionally hard to obtain owing to the lack of proper sources and sensors ${ }^{12,13}$. Notably, metamaterials induced extraordinary optical transmission (EOT) operating in the $\mathrm{THz}$ region has become an extremely active research area. It results in both exploring new methods of improving optical transmission and applying it to detecting, imaging, and nonlinear enhancement, among others ${ }^{14-16}$. Moreover, the capability of modulating the EOT effect is appealing for practical applications. Recently, a few efforts have been made in controlling the EOT effect in the $\mathrm{THz}$ region using metamaterials incorporated with different active mediums such as graphene ${ }^{17,18}$ and vanadium dioxide $\left(\mathrm{VO}_{2}\right)^{19,20}$. Graphene is a promising platform for $\mathrm{THz}$ modulators since it can obtain the electrically engineered density of states for intraband transition in the $\mathrm{THz}$ region ${ }^{21-23}$. Nevertheless, the one-atomic-layer thickness and low free carrier density of graphene limit the efficiency (i.e., extinction ratio) of these $\mathrm{THz}$ modulators, where a non-resonant nature of the intraband absorption exists ${ }^{24,25}$. Vanadium dioxide $\left(\mathrm{VO}_{2}\right)$ is also a promising candidate for tuning the EOT response from subwavelength apertures in a metal layer ${ }^{19,26}$ due to its phase transition from insulator to metal ${ }^{27,28}$. However, the practical suitability of $\mathrm{VO}_{2}$ for terahertz EOT metadevices is hampered by its volatility, complicated fabrication, and limitations of multilevel response and reversible phase transition ${ }^{29}$. Many works have demonstrated metamaterials based on the various active materials for actively controlling the EOT effect. Yet, to our knowledge, no EOT meta-devices presented to date have a combination of an ultrafast speed, a considerable modulation efficiency, nonvolatility, and repeatable reversible switching. It is, however, a highly important technique that may take a big stride forward towards practical applications.

An exciting new opportunity is suggested by recent developments in the chalcogenide phase change materi- als (PCMs) $)^{30,31}$. Chalcogenide PCMs such as Ge-Sb-Te alloys undergo phase transitions facilitated by nucleation dynamics $^{32}$; therefore, by continuously crystallizing the Ge-Sb-Te alloys, an analogue response can be obtained instead of binary switching states. Note that these analogue states are non-volatile; energy is only needed for the switching activity and not for maintaining an individual state ${ }^{33,34}$. Moreover, the Ge-Sb-Te alloys possess loads of desirable transiting features, such as short switching periods and high cyclability ${ }^{35}$. Thus this fuels the epoch of rewritable optical data storage and memory ${ }^{36,37}$. Furthermore, the fast, reversible, and nonvolatile amorphous-to-crystalline switching in the most studied Ge-Sb-Te alloy, $\mathrm{Ge}_{2} \mathrm{Sb}_{2} \mathrm{Te}_{5}$ (GST225), makes it ideal for reconfigurable photonic devices ${ }^{38-40}$. Very recently, nanostructured GST225 has been exploited to achieve specific switching properties ${ }^{41-43}$. The usability of GST225 for the different kinds of photonic devices is because that the dielectric properties of GST225 undergo a significant variation during its structural state change in the infrared region ${ }^{44,45}$. Since the radical contrast of optical property of the chalcogenide PCMs ranges from the IR to $\mathrm{THz}$ regions, it is promising for tunable $\mathrm{THz}$ metadevices. The chalcogenide PCM of GeTe has been studied previously for actively controlling the $\mathrm{THz}$ wave with nonvolatility ${ }^{46}$. Subsequently, a very recent work showed that $\mathrm{THz}$ chalcogenide metamaterials with a broken symmetry could realise a non-reversible tuning of Fano resonance by thermal annealing systems ${ }^{47}$. These works show that the chalcogenide PCMs can be valuable materials for obtaining actively tunable metamaterials in the $\mathrm{THz}$ regime. Yet, the versatile nature of the chalcogenide PCMs and their potential in the realisation of dynamically reconfigurable $\mathrm{THz}$ wave modulations have remained mostly undiscovered.

In this paper, we present an approach for the realization of dynamical, scalable, non-volatile, and repeatedly reconfigurable meta-devices to manipulate the EOT effect in the $\mathrm{THz}$ region. This metamaterial is composed of an array of subwavelength gold $\mathrm{(Au}$ ) holes that are fabricated on the GST225 switchable laminate. The EOT resonance results from the coupling of the $\mathrm{THz}$ wave with plasmons-electronic excitations-on the surface of the Au holes array. By optically changing the GST225 conductivity via an amorphous-crystalline state change, we accomplish switching on/off of the EOT with a modulation efficiency of intensity as high as $88 \%$. Furthermore, we experimentally show that such metamaterials 
can provide a very robust reconfigurable EOT effect over 20 times of transition. With the benefits of multiple-time reversible switching, high on/off ratios, simple geometry, and lightweight, our proposed meta-devices may be suitable for applications in diverse fields of $\mathrm{THz}$ sensing, communications, imaging, and so on.

\section{Results and discussion}

\section{Design and fabrication}

Figure 1(a) schematically shows the GST225-based THz modulator. The EOT THz metamaterials composed of Au holes array are placed above the $0.1 \mu \mathrm{m}$ thick GST225 layer sitting on the silicon ( $\mathrm{Si}$ ) substrate. The pitch of the metamaterials is $p=100 \mu \mathrm{m}$, while the diameter and depth of the Au holes are $d=60 \mu \mathrm{m}$ and $h_{\mathrm{Au}}=200 \mathrm{~nm}$, respectively. To avoid interlayer diffusion and interfacial reactions, a $5 \mathrm{~nm}$ thick silicon nitride $\left(\mathrm{Si}_{3} \mathrm{~N}_{4}\right)$ diffusion barrier was deposited between the Au holes and GST225 laminate ${ }^{48}$. The finite element method (FEM) is employed to solve Maxwell's equations for the metamaterial based on the amorphous GST225. We use the measured conductivity $(\sigma)$ of the GST225 film at room temperature (see a black line in Fig. 1(d)). The Au was modelled as a lossy metal with a conductivity of $4.1 \times 10^{7} \mathrm{~S} / \mathrm{m}$. All the metamaterials are modelled under normal plane wave illumination and the wave vector pointing towards the $+z$ direction, see Fig. 1(a). In Fig. 1(b), we numerically explored the dependence of the diameter and depth of the Au hole on the transmission of the chalcogenide metamaterials with amorphous state. In the top panel, we simulated the transmission for $h_{\mathrm{Au}}=200 \mathrm{~nm}$ at the different $d$. As was seen, the peak transmission increased with $d$ and tended to reduce when $d>60 \mu \mathrm{m}$. In the bottom panel, we numerically presented the transmission spectrum of the EOT metamaterials by varying the $h_{\mathrm{Au}}$ from $50 \mathrm{~nm}$ to $300 \mathrm{~nm}$ at $d=60 \mu \mathrm{m}$. As was seen, the peak transmission increased with the $h_{\mathrm{Au}}$ while it was maintained for $h_{\mathrm{Au}} \geq 100 \mathrm{~nm}$. It is because that the transmission spectrum of the EOT metamaterials was independent of the Au film thickness when it is larger than the skin depth in the $\mathrm{Au}^{49,50}$. Thus, herein the diameter and height of the Au holes were determined at $d=60 \mu \mathrm{m}$ and $h_{\mathrm{Au}}=200 \mathrm{~nm}$. The simplicity of this EOT metamaterial design is promising for applications in the $\mathrm{THz}$ integrated devices.

The EOT originates from excitation of the surface Bloch mode (SBM). Such a resonant SBM can enhance the field at the Au surface and, thus, the evanescent funneling of $\mathrm{THz}$ wave through the subwavelength holes array $^{51,52}$. Namely, the EOT was created by the multiple scatterings of flat-interface surface waves launched by each Au hole in the $\operatorname{array}^{53}$. Two different surface waves are involved, the surface plasmon polariton and quasicylindrical wave, which appear with a fixed mixing ratio to form a hybrid wave ${ }^{54,55}$. Particularly, in the EOT phenomenon, incident light is scattered by the Au subwavelength holes array into surface plasmons on the top surface of the Au. Subsequently, the surface plasmons tunnel through the Au holes array and excite surface plasmons on the bottom surface of the Au. Then, the surface plasmon on the bottom Au surface re-emits into free space, leading to a transmitted diffraction peak with a high peak intensity at the EOT resonance frequency ${ }^{56-58}$. The subwavelength Au holes play an essential role in the EOT effect through optical coupling between the surface plasmons on the top and bottom $\mathrm{Au}$ surfaces. Consequently, the total electric ( $E$-) field is significantly enhanced around and inside the Au subwavelength holes, exciting the EOT phenomenon.

In our proposed modulator, GST225 film is placed below the Au holes to engineer the EOT resonant coupling. This is achieved by modulating the conductivity of GST225 via its phase transition between amorphous and crystalline. For the amorphous state, the GST225 film possesses a minimal conductivity and is transparent at the operating frequency, allowing for a high transmission of $\mathrm{THz}$ wave at the EOT resonance. For the crystalline state, the conductivity of GST225 significantly increases to block the wave transmission at the EOT resonance. Thus, an intense suppression of EOT appears. To illustrate this tuning mechanism, we fabricated the subwavelength Au nanoholes array on the GST225 membrane, and the optical microscope image of the structure was presented in the top panel of Fig. 1(c). A $100 \mathrm{~nm}$ thick GST225 laminate is first fabricated onto a $1 \mathrm{~mm}$ thick silicon ( $\mathrm{Si}$ ) substrate. The Au holes array is made using standard photolithography techniques and electron beam deposition of $200 \mathrm{~nm}$ of $\mathrm{Au}$, followed by a liftoff procedure. A flowchart of the entire fabrication process is schematically illustrated in Supplementary information Fig. S1. The sample has a relatively large array area of $4 \mathrm{~cm} \times 4 \mathrm{~cm}$ so that a focused THz beam can fit. A focused ion beam (FIB) cross section of the metamaterials, which is presented in bottom panel of Fig. 1(c), can be employed to find the accurate thickness of each layer 


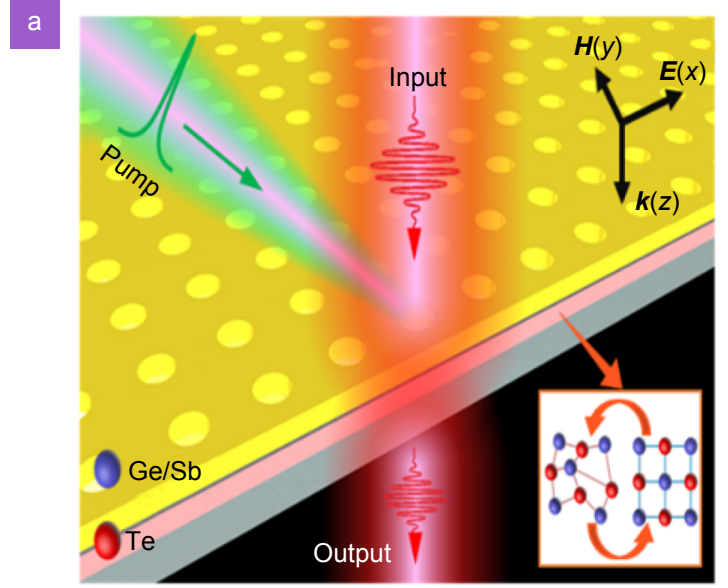

c
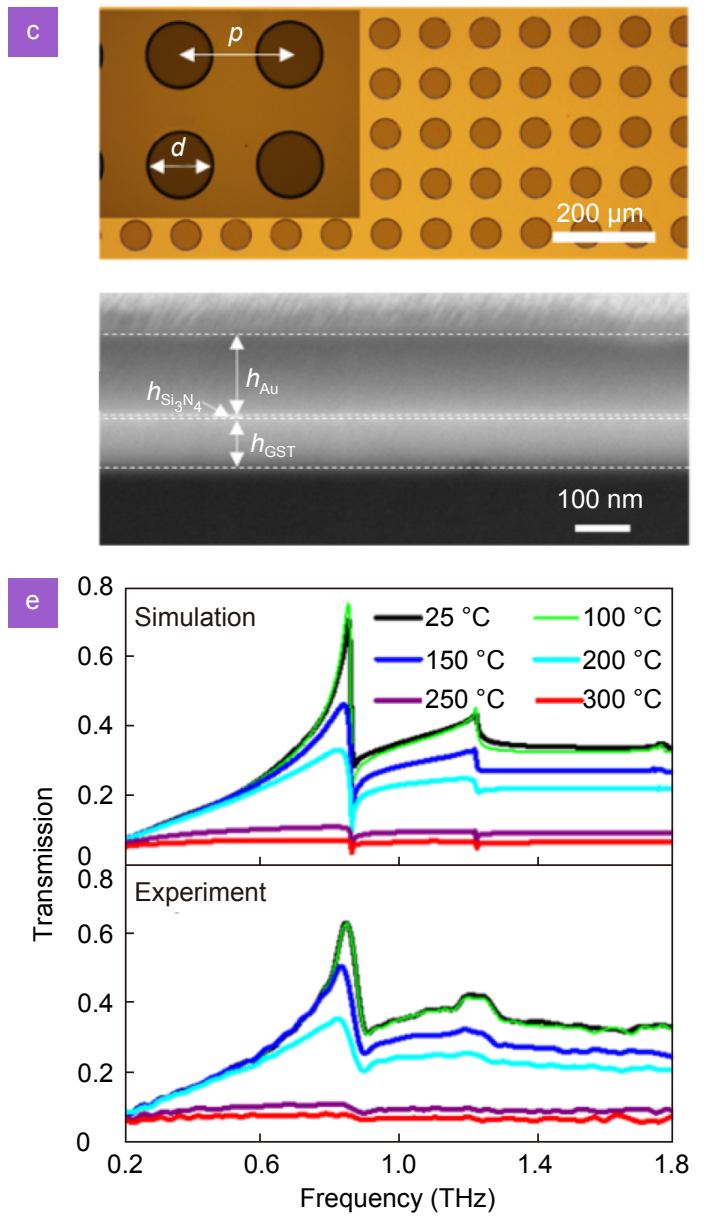

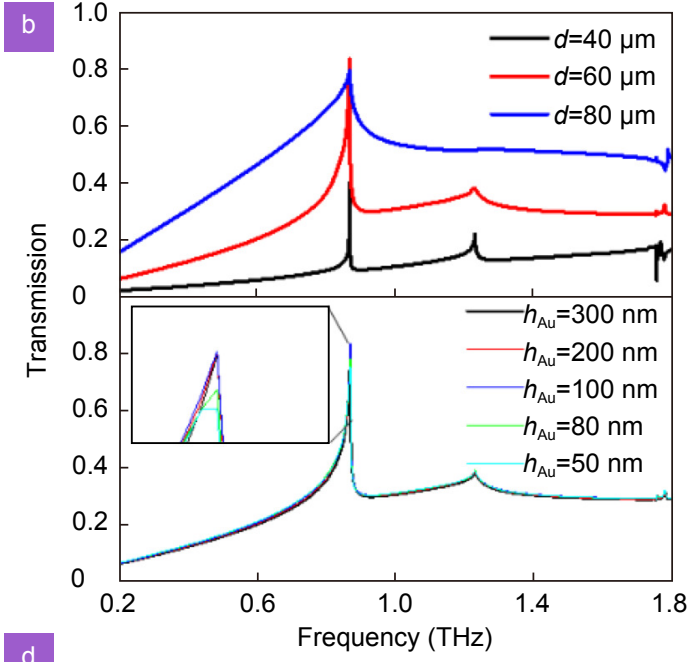

d
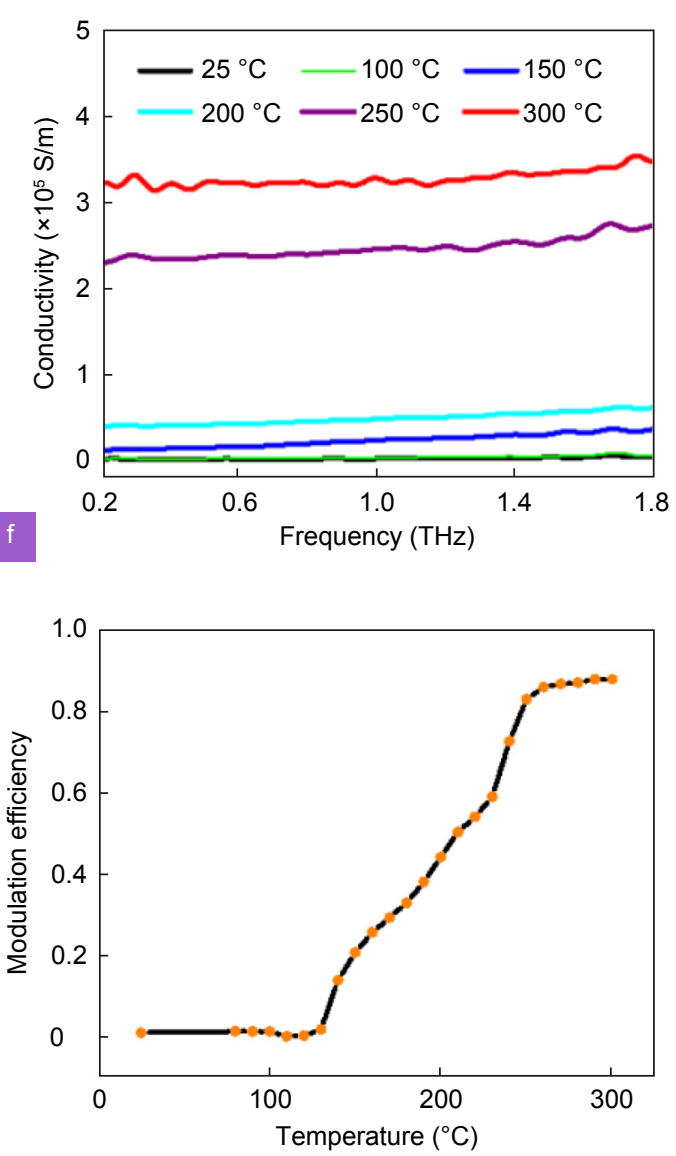

Fig. 1 | Configuration of the EOT metamaterials for the THz region. (a) Schematic of the all-optical, reconfigurable, non-volatile phasechange metamaterials induced EOT switch: single nanosecond pulsed laser transits a $100 \mathrm{~nm}$ thick GST225 film reversibly between the amorphous and crystalline states. (b) The FEM simulated transmission spectra of the chalcogenide metamaterials with the amorphous state at the various diameter of $d=40,60$, and $80 \mu \mathrm{m}$ (top panel) and various heights of $h_{\mathrm{Au}}=50,80,100,200$, and $300 \mathrm{~nm}$ (bottom panel). (c) Optical microscope (top panel) and FIB cross-sections (bottom panel) images of the EOT metamaterials. The geometrical parameters of the subwavelength holes array are $p=100 \mu \mathrm{m}, d=60 \mu \mathrm{m}$, respectively; the thicknesses of the Au and GST225 layers are $h_{\mathrm{Au}}=0.2 \mu \mathrm{m}$ and $h_{\mathrm{GST}}=0.1 \mu \mathrm{m}$, respectively. (d) The temperature-dependent optical conductivity $(\sigma)$ of the $100 \mathrm{~nm}$ thick GST225 film. (e) The behavior of resonant transition in the metamaterials: numerical simulated (top panel) and experimental measured (bottom panel) transmission spectra at the various temperatures ranging from $25^{\circ} \mathrm{C}$ to $300^{\circ} \mathrm{C}$. The reduction of the peak intensity can be experimentally and theoretically observed by increasing the temperature. (f) The modulation efficiency against the annealing temperature varied from $25^{\circ} \mathrm{C}$ to $300^{\circ} \mathrm{C}$. 
of the metamaterials. As was seen, the measured thicknesses of $\mathrm{Au}, \mathrm{Si}_{3} \mathrm{~N}_{4}$, and GST225 films are 200, 5, and $100 \mathrm{~nm}$, respectively.

The optical conductivity $(\sigma)$ of $100 \mathrm{~nm}$ thick GST225 film in the $\mathrm{THz}$ region is extracted from the transmitted $\mathrm{THz}$ time signal through the GST225 film with the Si substrate as a reference. Fig. 1(d) presents the temperature dependent $\sigma$. By thermally crystallizing the as-deposited (AD) amorphous (AM) GST225 film, the $\sigma$ possesses a pronounced contrast and is weakly dispersive over the $\mathrm{THz}$ region. A large increase in the $\sigma$ is found with a three increasing order as transiting the GST225 state from AD-AM to crystalline (CR). For example, the $\sigma$ of AD-AM GST225 film is $\sim 108 \mathrm{~S} / \mathrm{m}$ (black line) which is boosted to $\sim 321868 \mathrm{~S} / \mathrm{m}$ for $300{ }^{\circ} \mathrm{C}$ annealed CR film (red line). Such a remarkable change in the $\sigma$ between the AD-AM (black line) and CR (red line) states can switch the EOT response "on/off" at a fixed THz frequency. The large difference of $\sigma$ in GST225 originates from bonding switching between the predominantly covalent bonds in the AM state and resonant bonds in the CR state ${ }^{30,40}$. Namely, the CR- GST225 has an extremely large $\sigma$, resulting from highly polarisable delocalized p-orbital resonant bonding. The AM-GST225 that is built from the predominantly covalent bonds has a much lower $\sigma$. Note that the GST225 film is gradually crystallised, which can form an intermediate state exhibiting regions of both amorphous and crystalline phases ${ }^{59}$. By controlling the annealing time and temperature, the crystalline fraction in the AD-AM GST225 film can be finely modified, leading to a gradual variation in the $\sigma$. Such an attractive feature can offer a more continuous modulation of the EOT effect in the proposed $\mathrm{THz}$ metamaterials. To show this, the measured $\sigma$ of the GST225 film at the different heating temperatures is also demonstrated in Fig. 1(d). As can be seen, the $\sigma$ increases with the temperature in the spectral range from 0.2 to $1.8 \mathrm{THz}$. Note, the largest change in the $\sigma$ appears when increasing the temperature from 200 to $300{ }^{\circ} \mathrm{C}$. Importantly, these intermediate states of GST225 are non-volatile. Therefore, they are very stable at room temperature, and the thermal energy is only required for switching the structural state, not for maintaining a certain state. This makes the continuously tunable $\mathrm{THz}$ EOT meta-device appealing from a green technology perspective.

The FEM simulated transmission spectra under the different temperatures are shown in the top panel of Fig. $1(\mathrm{e})$. We use the measured temperature-dependent conductivity $(\sigma)$ of the GST225 film that is shown in Fig. 1(d). The metamaterial geometry is set to that measured using the optical microscope and FIB cross-section images presented in Fig. 1(c). A decrease of the resonant peak occurs by increasing the temperature. The transmission $(T)$ of the fabricated $\mathrm{THz}$ chalcogenide metamaterials under the different temperatures, from $25{ }^{\circ} \mathrm{C}$ to $300{ }^{\circ} \mathrm{C}$ are measured by $\mathrm{THz}$ time-domain spectroscopy (THz-TDS) system. The THz time-domain data are temporally windowed to remove the effect of multiple reflectances within the Si substrate. The measured transmission spectra are shown in the bottom panel of Fig. $1(\mathrm{e})$. The transmission spectra are polarisation independent due to the structural symmetry. As can be seen, the peak transmission decreases with the temperature, while the central frequency remained almost the same at different temperatures. For the AD-AM state, the EOT resonance is measured at $f_{1}=0.85 \mathrm{THz}$ and reaches the maximum value of $T=0.63$ (black line) due to the tiny $\sigma$ of GST225 film. Note that starting with the $T_{\mathrm{C}}=250{ }^{\circ} \mathrm{C}$ measurement (purple line), the GST225 film is crystallized to obtain a high $\sigma$, thus demonstrating a metallic behavior. This is manifest by the radical variation in the transmission spectra between the measurements taken at $200{ }^{\circ} \mathrm{C}$ (cyan line) and $250{ }^{\circ} \mathrm{C}$ (purple line). When thermally annealed to $300{ }^{\circ} \mathrm{C}$, the EOT resonance is entirely turned off (red line). The numerically calculated transmission spectra under the different temperatures have a good agreement with the measured ones. The FEM simulations (top panel) slightly differ from the experimental measurements (bottom panel) in a few ways. The simulated modulation of EOT intensity is higher than the experimental one, and the bandwidth of the measured resonance is significantly wider. These characteristics are owing to certain factors that differentiate simulations from measurements. The incident light is lightened by a Cassegrain-type microscope objective lens possessing a numerical aperture (NA) of 0.58 , showing the angle of incident light ranges from $-35^{\circ}$ to $+35^{\circ}$. Such a wide angular distribution of incident light leads to a lower transmission in the EOT resonance and a wider transmission spectrum compared to the simulated one. This is because the EOT resonance is strongly associated with the incident angle. Besides, in the model, we ignore the fabrication imperfections, surface roughness and native oxides. We presume this simplification causes the slight broadening of the experimentally measured spectra and the reduction of the modulation efficiency compared to the numerical calculation. We then demonstrate how the metamaterial transmission spectra can be 
actively controlled by transiting the state of the GST225 via the temperature variation. This capability to cover a portion of the $\mathrm{THz}$ region by gradually crystallising the GST225, is the core of the $\mathrm{THz}$ modulator.

In Fig. 1(f), we have evaluated the modulation performance of the meta-devices. Herein, the modulation efficiency $\left(\zeta_{\mathrm{m}}\right)$ in the transmission is defined by $\zeta_{\mathrm{m}}=1-$ $T / T_{\max }$, where transmission $T$ is dependent on the temperature, and $T_{\max }$ is the peak transmission for the $\mathrm{AD}$ AM metamaterials that exhibit the most substantial transmitted intensity at the resonant frequency. The $\zeta_{\mathrm{m}}$ increases with the temperature. For instance, at the room temperature of $25^{\circ} \mathrm{C}$, the modulation efficiency of the metamaterials is $\zeta_{\mathrm{m}}=0$, while the EOT metamaterials can achieve $\zeta_{\mathrm{m}}=88 \%$ at the temperature of $300{ }^{\circ} \mathrm{C}$. Therefore, the EOT metamaterials can possess a significant modulation in transmission by switching the state of the GST225 between amorphous and crystalline states. Modulation efficiency is a crucial parameter for evaluating the performance of the modulators. In the last dec-
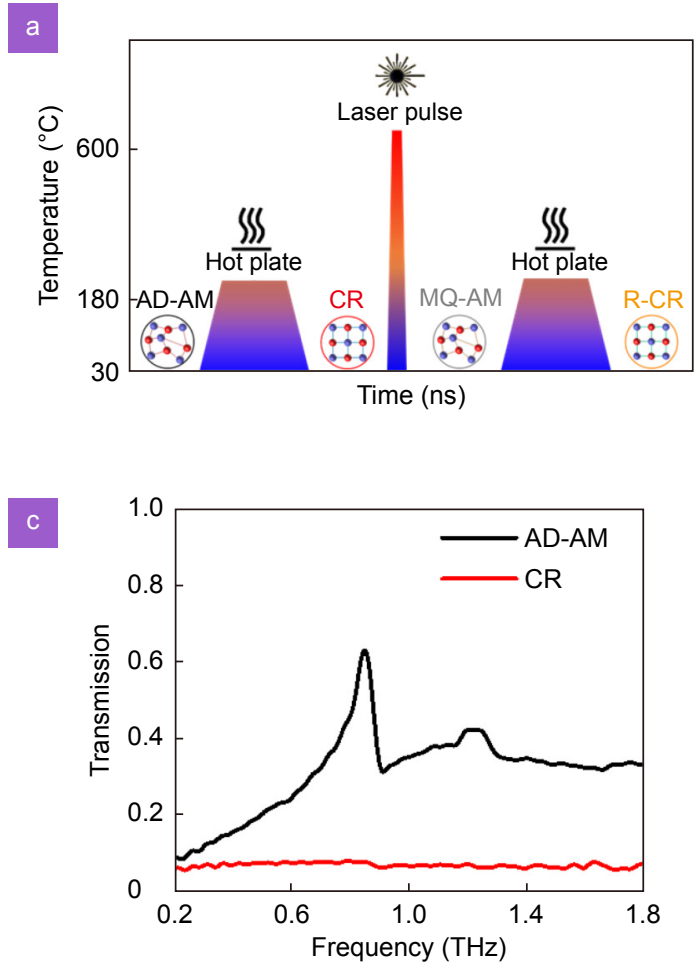

ade, the THz modulators have developed rapidly, and the modulation efficiency has increased from $40 \%$ to nearly $90 \%{ }^{60}$. Our proposed chalcogenide metamaterials with a high modulation efficiency of $88 \%$ may build up a platform for developing new kinds of $\mathrm{THz}$ modulators (i.e., compressing sensor modulator and on-chip modulator $)^{61}$, which provides an extraordinary function for manipulating $\mathrm{THz}$ waves.

\section{Experimental realization of reconfigurable EOT}

The GST225 film exhibits crystallization and melting temperatures of $T_{\mathrm{C}}=250^{\circ} \mathrm{C}$ and $T_{\mathrm{M}}=600^{\circ} \mathrm{C}$, respectively. It has been highly developed as a platform for efficient, non-volatile, prompt, reproducible state switching ${ }^{62,63}$. Herein, the alternation between the amorphous and crystalline structural phases offers a significant change in the $\sigma$ of GST225 film, which subsequently modulates the EOT resonant intensity. The amorphisation of GST225 can be experimentally realised by the illumination of a nanosecond (ns) pulsed laser,

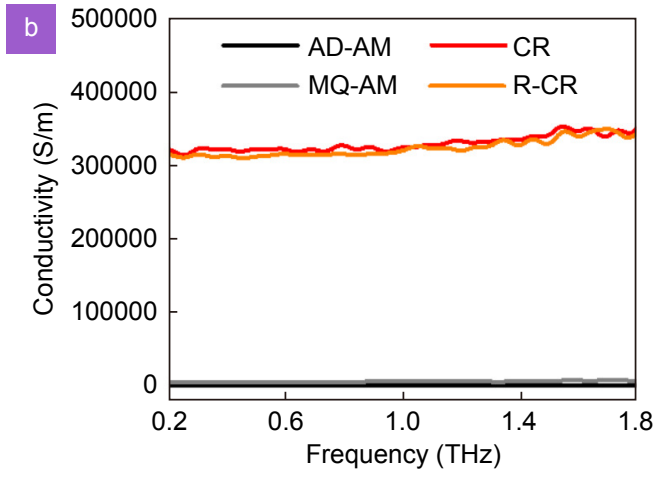

d

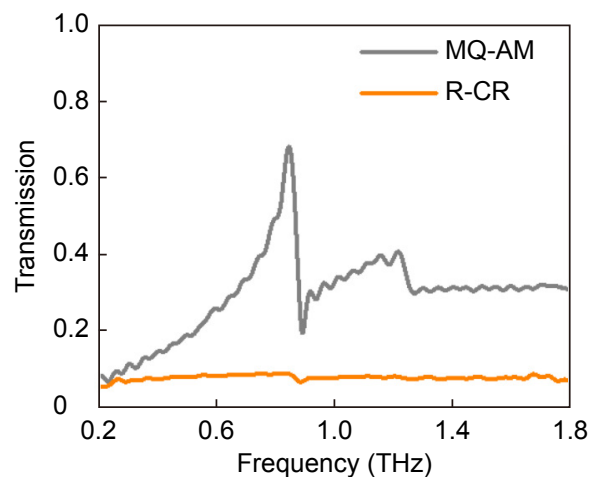

Fig. 2 | In sequence processing for the reversible state change. (a) Schematic of the reversible state change of the GST225 layer hybridised with an EOT metamaterials: the AD-AM GST225 is initially heated above $T_{\mathrm{C}}=250{ }^{\circ} \mathrm{C}$ to switch to the CR-GST225 via a hot plate. A single ns pulsed laser is transited to thermally anneal the CR-GST225 layer above $T_{\mathrm{M}}=600^{\circ} \mathrm{C}$ that reamorphises the CR-GST225. Consequent quenching leads to the MQ-AM GST225. A temperature above $T_{\mathrm{C}}=250^{\circ} \mathrm{C}$ but below $T_{\mathrm{M}}=600{ }^{\circ} \mathrm{C}$ is needed to recrystallise the MQ-AM GST225, which is achieved by using a hot plate. (b) The $\sigma$ of $100 \mathrm{~nm}$ thick GST225 film at the various structural states of the as-deposited amorphous (AD-AM, black line), crystalline ( $C R$, red line), melt quenched amorphous (MQ-AM, grey line), and re-crystallised (R-CR, orange line) over a spectral range of 0.2-1.8 THz. Experimental realisation of reversibly tunable EOT effect: the THz-TDS measurement of the transmission spectra of the chalcogenide metamaterials with the various structural phases of (c) AD-AM, CR and (d) MQ-AM, and R-CR. 
while the recrystallisation of GST225 can be realised by the hot plate ${ }^{64,65}$. In Fig. 2(a), we schematically show the phase change metamaterials treatment. First, the ADAM GST225 film is crystallised by heating the metamaterials for 30 mins at $T_{\mathrm{C}}=250^{\circ} \mathrm{C}$ on a hot-plate in a flowing $\mathrm{Ar}$ atmosphere. To reversibly transit the structural state of the GST225 film from CR to MQ-AM, the crystal lattice is melted and then quenched into the AM state (room temperature) under a rate of $10^{9} \sim 10^{10} \mathrm{~K} / \mathrm{s}$ that prevents recrystallisation of the atomic structure ${ }^{66,67}$. Ultrashort laser Joule pulses can provide such a quench rate $^{68}$, which is employed to switch the $100 \mathrm{~nm}$ thick GST225 film from the CR to AM states. Particularly, to amorphise the crystalline GST225 laminate, a single $5 \mathrm{~ns}$ laser pulse makes the local temperature momentarily increase above $600{ }^{\circ} \mathrm{C}$ to melt the GST225. A fast-cooling processing is subsequently performed to quench the melted layer into the AM phase. To recrystallise the MQAM GST225 layer, a hot plate is used to hold the GST225 film above $T_{\mathrm{C}}=250{ }^{\circ} \mathrm{C}$ but below $T_{\mathrm{M}}=600{ }^{\circ} \mathrm{C}$. The phase transition (amorphous to crystalline) of GST225 can also be electrically achieved by integrating a bottom metallic heater to the metasurfaces, as schematically shown in Supplementary information Fig. S2.

Figure 2(b) shows the $\sigma$ of a $100 \mathrm{~nm}$ thick monolithic GST225 layer for the AD-AM (black line), CR (red line), MQ-AM (grey line), and re-crystallised (R-CR, orange line) structural phases. Note, the spectra of the optical properties $(\sigma)$ for AD-AM and MQ-AM GST225, as well as for CR and R-CR GST225, are almost identical. It confirms that the GST225 phase transition can be reversible, and the material after the ultrashort pulsed laser-induced change resembles the initial states after hot-plate annealing. In Fig. 2(c)-2(d), we demonstrate experimentally a reconfigurable metamaterial modulator of EOT operating at the $\mathrm{THz}$ frequency using a reversible phase transition between the AM and CR in the GST225 film. The transmission spectra are divided into two sections to distinguish between the metamaterial treatments: a comparison between the AD-AM (black line) and thermally annealed CR (red line) states is presented in Fig. 2(c), and the comparison between the MQ-AM (grey line) and R-CR (orange line) states after ns laserinduced phase transition is shown in Fig. 2(d). When the GST225 is in the AD-AM and MQ-AM states, the EOT resonant peak with a transmission magnitude of 0.63 at $f_{1}=0.85 \mathrm{THz}$ occurs. When the GST225 is crystallised by thermal annealing (CR and $\mathrm{R}-\mathrm{CR})$, the EOT resonance vanishes. This is because the $\sigma$ of the CR- GST225 is much larger than that of the AM-GST225 (Fig. 2(b)), which increases the loss to decrease the peak intensity and broaden the bandwidth of the EOT phenomenon. This produces a tremendous modulation efficiency at $f_{1}=0.85 \mathrm{THz}$. The active transmission engineering in the meta-device possesses a clear advantage over the previously shown passive THz EOT metamaterials.

\section{Resonant modes in EOT}

The origin of the EOT effect is based upon the strong enhancement of the total electrical field intensity $\left(|\boldsymbol{E}|^{2}=\left|E_{x}\right|^{2}+\left|E_{y}\right|^{2}+\left|E_{z}\right|^{2}\right)$ inside the Au subwavelength holes array. In order to observe this origin, it is helpful to investigate the distributions of the total $E$-field intensities of the metamaterials at the different temperatures along the $x-z$ cross-section plane. Fig. 3 presents the $E$ field distributions at the resonant frequencies of 0.87 $\mathrm{THz}$ corresponding to the various temperatures of $25^{\circ} \mathrm{C}$, $150{ }^{\circ} \mathrm{C}, 200{ }^{\circ} \mathrm{C}$, and $300{ }^{\circ} \mathrm{C}$, respectively. In the profile, the colour represents the magnitude of the $E$-field intensities. The $\mathrm{THz}$ wave is incident from the top of the metamaterials. It demonstrates that the EOT effect can be interpreted by a cavity resonance. The total $E$-field intensity distribution for the amorphous state is shown in Fig. 3(a). It clearly shows that the total $E$-field can couple in the GST225 film and is intensely enhanced around and inside the hole region, which is a feature of a typical EOT effect. In Fig. 3(b)-3(d), the total E-field intensity distributions at $f=0.87 \mathrm{THz}$ for the various temperatures of $150{ }^{\circ} \mathrm{C}, 200{ }^{\circ} \mathrm{C}$, and $300^{\circ} \mathrm{C}$ are explored. It can be seen that with an increase of the temperature, the EOT resonance is largely damped and hence suppresses the total $E$-field inside the holes.

\section{Repeatedly rewritable experiments}

To examine the repeatability of optical switching of the EOT metamaterial, we repeatedly performed rewritable experiments. Fig. 4(a) experimentally illustrates the transmission spectra of the EOT metamaterials for twenty transition times. As can be seen, the metamaterials give rise to a very efficient reconfigurable EOT effect over many times of switching, taking advantage of a diffusion barrier of $\mathrm{Si}_{3} \mathrm{~N}_{4}$ between the Au holes and the GST225 film. Fig. 4(b) presents the values of the peak transmission for amorphous (black dots) and crystalline (red dots) states with twenty switching times. Herein, the amorphous GST225 film can be crystallised by thermally 

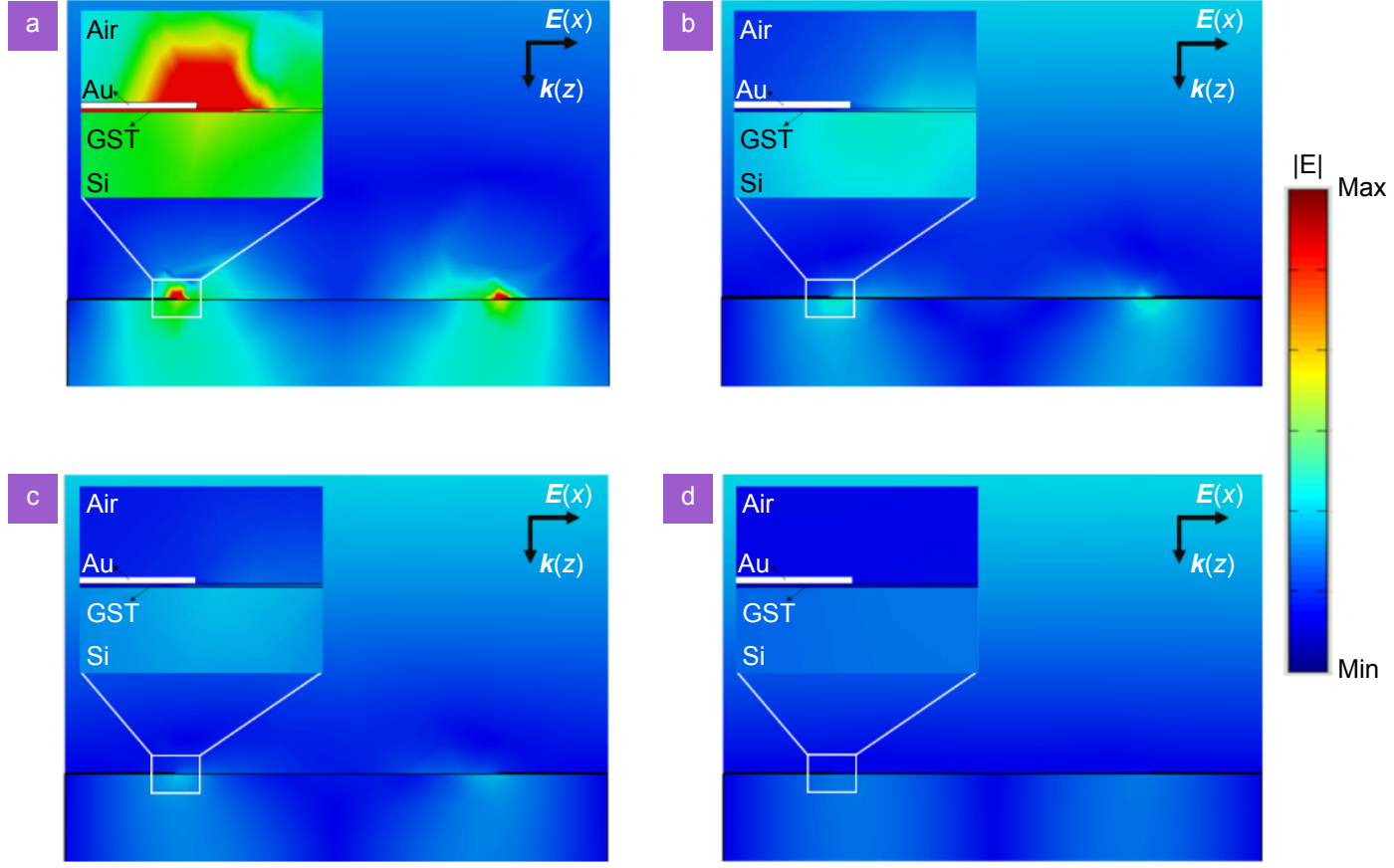

Fig. 3 | Numerical simulation of total $E$ - field distribution along the $x$-z plane of the metamaterials at the various temperatures of (a) $25^{\circ} \mathrm{C}$, (b) 150 ${ }^{\circ} \mathrm{C}$, (c) $200^{\circ} \mathrm{C}$, and (d) $300^{\circ} \mathrm{C}$.
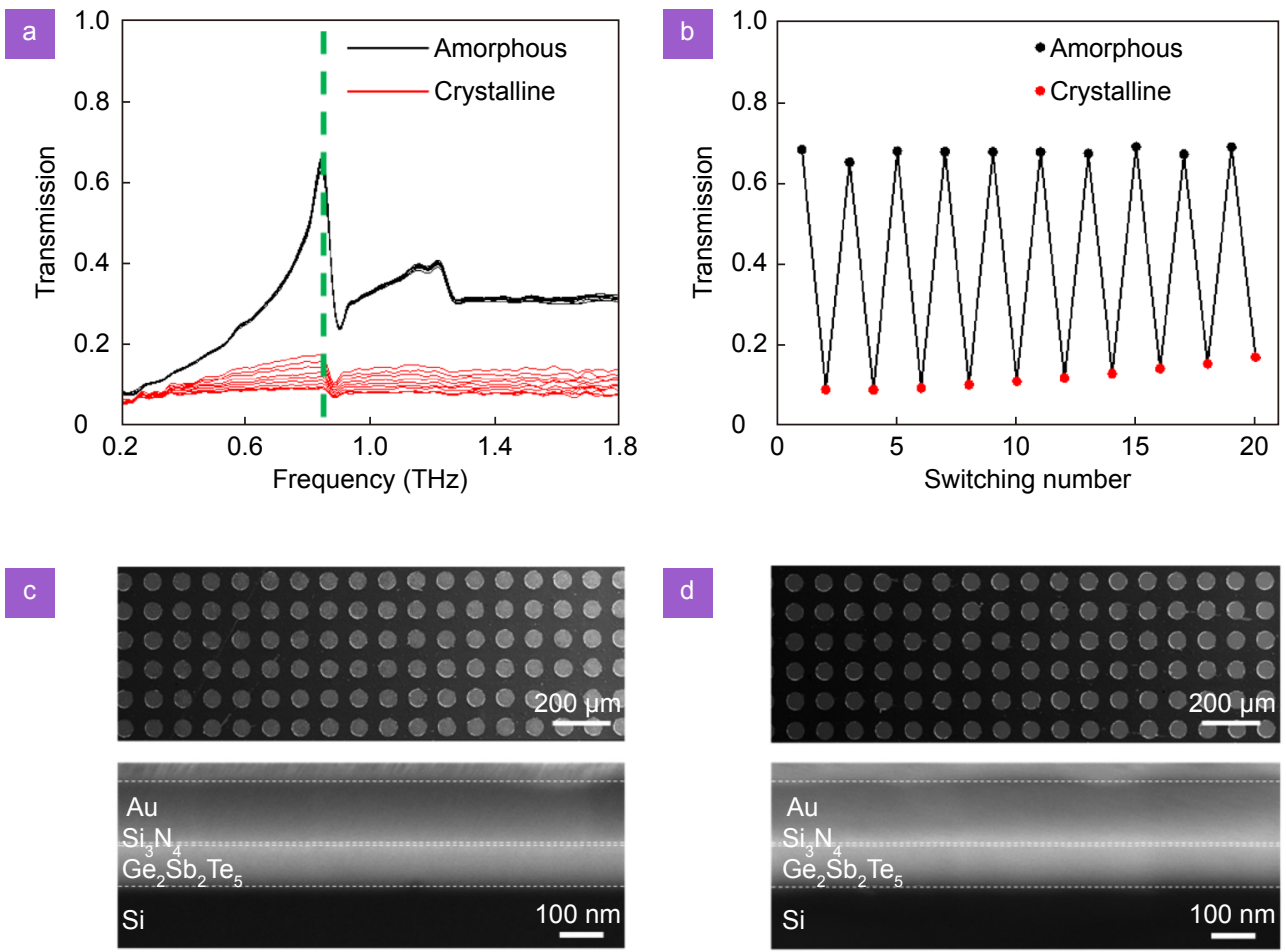

Fig. 4 | (a) Measured transmission spectra of the EOT chalcogenide metamaterials for twenty switching times. (b) The values of transmission peaks for the amorphous (shown by black dots) and crystalline (indicated by red dots) states with twenty switching times. The morphology (top panel) and cross-sectional (bottom panel) images of the chalcogenide metamaterials (c) before and (d) after 20 transition times.

annealing the metamaterials for $3 \mathrm{~min}$ at $T=300{ }^{\circ} \mathrm{C}$ on a hotplate. To amorphise the crystalline GST225 film, an ultrafast laser pulse was employed to momentarily increase the temperature of GST225 film above $600{ }^{\circ} \mathrm{C}$ and then cool promptly down into the room temperature. The ultrafast laser pulse has a central wavelength of 1064 $\mathrm{nm}$, pulse width of $5 \mathrm{~ns}$, a spot diameter of $1 \mathrm{~cm}$, and peak fluence of $105 \mathrm{~mJ} / \mathrm{cm}^{2}$. Such a rewritable experiment 
was cycled to achieve the repeatable optical switching of the EOT response.

In the proof-of-principle metamaterials experimentally realised here, the functional GST225 layer typically endures $\approx 10$ switching cycles (amorphous-to-crystalline and backward) before demonstrating the degradation sign. It is induced by degradation of the GST225 due to the formation of voids. Initially, the tiny voids with a few nanometers size were distributed inside the crystalline GST225 layer. After more repeated switching cycles, the sizes of voids have become larger ${ }^{69}$. This leads to gradual segregation of the elements of $\mathrm{Ge}, \mathrm{Sb}$, and $\mathrm{Te}$ in the various regions of the device. Such segregation can slowly change the switching features of the device ${ }^{70}$. Moreover, the atomic contents of $\mathrm{Ge}, \mathrm{Sb}$, and Te varied after repeated heating around the voids ${ }^{71}$, enabling the compositional variations to be closely associated with the void formation in the GST225 layer. These compositional changes may reduce endurance characteristics during the metadevice operations ${ }^{72-74}$. It is expected that the device robustness can be improved by optimizing the compositions and thicknesses of GST225 film. The melting point temperatures of silicon $(\mathrm{Si})$ and silicon nitride $\left(\mathrm{Si}_{3} \mathrm{~N}_{4}\right)$ are $T_{\mathrm{Si}}=1410{ }^{\circ} \mathrm{C}$ and $T_{\mathrm{SiN}}=1990{ }^{\circ} \mathrm{C}$, respectively ${ }^{75}$, which are much higher than the melting temperatures $\left(T_{\mathrm{M}}=600{ }^{\circ} \mathrm{C}\right)$ of GST225 film. Therefore, the Si and $\mathrm{Si}_{3} \mathrm{~N}_{4}$ films were not significantly affected after 20 transition times. Figure 4(c) and 4(d) present the FIB images of the chalcogenide metamaterials before and after 20 transition times, where the morphology and side view of the metamaterials were shown in top and bottom panels, respectively. As was observed, the thickness of each layer and morphology of the Au holes array can be maintained during the phase transition of GST225. Moreover, by integrating the GST225 active component into the various functional resonators such as split-ring ${ }^{76,77}$, and $\mathrm{H}$-shaped ${ }^{78}$ resonators, one may also reversibly tune the resonant wavelength of the $\mathrm{THz}$ meta-devices. Beyond the reconfigurable EOT phenomenon, the GST225 based metamaterials may also find interesting applications for reconfigurable extraordinary optical diffraction response $^{79,80}$.

\section{Conclusions}

In conclusion, we have demonstrated a reconfigurable EOT metamaterial for the $\mathrm{THz}$ region, realised by ns laser-induced switching of the structural phase of the chalcogenide glass, GST225. The chalcogenide metama- terials provide non-volatile and active controlling of the EOT resonant mode by transiting the state of GST225 between amorphous and crystalline. Such a phase change leads to a large contrast in the $\sigma$, changing from $108 \mathrm{~S} / \mathrm{m}$ to $321868 \mathrm{~S} / \mathrm{m}$, which excites the EOT for the amorphous state, whereas suppresses the EOT for the crystalline state, causing a very high modulation efficiency of $88 \%$ at $0.85 \mathrm{THz}$. Importantly, the EOT effect can be reversibly tuned over 20 times of switching. These findings may offer chances in designing an on-chip $\mathrm{THz}$ wave modulator with a high on/off ratio, low power, high speed, and tailored operating frequency, opening up a range of state-of-the-art applications for $\mathrm{THz}$ imaging, communications, and sensing.

\section{References}

1. Tonouchi M. Cutting-edge terahertz technology. Nat Photonics 1, 97-105 (2007).

2. Zaytsev KI, Kudrin KG, Karasik VE, Reshetov IV, Yurchenko SO. In vivo terahertz spectroscopy of pigmentary skin nevi: pilot study of non-invasive early diagnosis of dysplasia. Appl Phys Lett 106, 053702 (2015).

3. Ma FS, Lin YS, Zhang $\mathrm{XH}$, Lee $\mathrm{C}$. Tunable multiband terahertz metamaterials using a reconfigurable electric split-ring resonator array. Light Sci App/ 3, e171 (2014).

4. Tong MY, Hu YZ, Xie XN, Zhu XG, Wang ZY et al. Helicity-dependent $\mathrm{THz}$ emission induced by ultrafast spin photocurrent in nodal-line semimetal candidate $\mathrm{Mg}_{3} \mathrm{Bi}_{2}$. Opto-Electron $\mathrm{Adv} \mathbf{3}$, 200023 (2020).

5. Schurig D, Mock JJ, Justice BJ, Cummer SA, Pendry JB et al. Metamaterial electromagnetic cloak at microwave frequencies. Science 314, 977-980 (2006).

6. Nemati A, Wang Q, Hong $\mathrm{MH}$, Teng JH. Tunable and reconfigurable metasurfaces and metadevices. Opto-Electron Adv 1, 180009 (2018)

7. Wang YL, Fan QB, Xu T. Design of high efficiency achromatic metalens with large operation bandwidth using bilayer architecture. Opto-Electron Adv 4, 200008 (2021).

8. Guo JY, Wang T, Quan BG, Zhao H, Gu CZ et al. Polarization multiplexing for double images display. Opto-Electron Adv 2, 180029 (2019).

9. Ma XL, Pu MB, Li X, Guo YH, Luo XG. All-metallic wide-angle metasurfaces for multifunctional polarization manipulation. Opto-Electron Adv 2, 180023 (2019).

10. Rahmani M, Leo G, Brener I, Zayats AV, Maier SA et al. Nonlinear frequency conversion in optical nanoantennas and metasurfaces: materials evolution and fabrication. Opto-Electron Adv 1, 180021 (2018).

11. Wang YQ, Ma XL, Li X, Pu MB, Luo XG. Perfect electromagnetic and sound absorption via subwavelength holes array. OptoElectron Adv 1, 180013 (2018).

12. Linden S, Enkrich C, Wegener M, Zhou JF, Koschny $\mathrm{T}$ et al. Magnetic response of metamaterials at 100 terahertz. Science 306, 1351-1353 (2004).

13. Yen TJ, Padilla WJ, Fang N, Vier DC, Smith DR et al. Terahertz magnetic response from artificial materials. Science $\mathbf{3 0 3}$, 
1494-1496 (2004).

14. Rodrigo SG, de León-Pérez F, Martín-Moreno L. Extraordinary optical transmission: fundamentals and applications. Proc IEEE 104, 2288-2306 (2016).

15. Padilla WJ, Aronsson MT, Highstrete C, Lee M, Taylor AJ et al. Electrically resonant terahertz metamaterials: theoretical and experimental investigations. Phys Rev B 75, 041102 (2007).

16. Bingham $\mathrm{CM}$, Tao H, Liu XL, Averitt RD, Zhang $X$ et al. Planar wallpaper group metamaterials for novel terahertz applications. Opt Express 16, 18565-18575 (2008).

17. Kim S, Jang MS, Brar VW, Tolstova Y, Mauser KW et al. Electronically tunable extraordinary optical transmission in graphene plasmonic ribbons coupled to subwavelength metallic slit arrays. Nat Commun 7, 12323 (2016).

18. Gao WL, Shu J, Reichel K, Nickel DV, He XW et al. High-contrast terahertz wave modulation by gated graphene enhanced by extraordinary transmission through ring apertures. Nano Lett 14, 1242-1248 (2014).

19. Seo M, Kyoung J, Park H, Koo S, Kim HS et al. Active terahertz nanoantennas based on $\mathrm{VO}_{2}$ phase transition. Nano Lett $\mathbf{1 0}$, 2064-2068 (2010).

20. Hendry E, Lockyear MJ, Rivas JG, Kuipers L, Bonn M. Ultrafast optical switching of the $\mathrm{THz}$ transmission through metallic subwavelength hole arrays. Phys Rev B 75, 235305 (2007).

21. Lee SH, Choi M, Kim TT, Lee S, Liu M et al. Switching terahertz waves with gate-controlled active graphene metamaterials. Nat Mater 11, 936-941 (2012).

22. Kim TT, Oh SS, Kim HD, Park HS, Hess O et al. Electrical access to critical coupling of circularly polarized waves in graphene chiral metamaterials. Sci Adv 3, e1701377 (2017).

23. Miao ZQ, Wu Q, Li X, He Q, Ding K et al. Widely tunable terahertz phase modulation with gate-controlled graphene metasurfaces. Phys Rev X 5, 041027 (2015).

24. Ju L, Geng BS, Horng J, Girit C, Martin M et al. Graphene plasmonics for tunable terahertz metamaterials. Nat Nanotechnol 6 , 630-634 (2011).

25. Thareja V, Kang JH, Yuan HT, Milaninia KM, Hwang HY et al. Electrically tunable coherent optical absorption in graphene with ion gel. Nano Lett 15, 1570-1576 (2015).

26. Seo MA, Park HR, Koo SM, Park DJ, Kang JH et al. Terahertz field enhancement by a metallic nano slit operating beyond the skin-depth limit. Nat Photonics 3, 152-156 (2009).

27. Driscoll T, Kim HT, Chae BG, Kim BJ, Lee YW et al. Memory metamaterials. Science 325, 1518-1521 (2009).

28. Zhao YC, Zhang YX, Shi QW, Liang SX, Huang WX et al. Dynamic photoinduced controlling of the large phase shift of terahertz waves via vanadium dioxide coupling nanostructures. ACS Photonics 5, 3040-3050 (2018).

29. Cai HL, Chen S, Zou CW, Huang QP, Liu Y et al. Multifunctional hybrid metasurfaces for dynamic tuning of terahertz waves. Adv Opt Mater 6, 1800257 (2018).

30. Shportko K, Kremers S, Woda M, Lencer D, Robertson J et al. Resonant bonding in crystalline phase-change materials. Nat Mater 7, 653-658 (2008).

31. Simpson RE, Fons $\mathrm{P}$, Kolobov $\mathrm{AV}$, Fukaya $\mathrm{T}$, Krbal $\mathrm{M}$ et al. Interfacial phase-change memory. Nat Nanotechnol 6, 501-505 (2011).

32. Jeong TH, Kim MR, Seo H, Kim SJ, Kim SY. Crystallization behavior of sputter-deposited amorphous $\mathrm{Ge}_{2} \mathrm{Sb}_{2} \mathrm{Te}_{5}$ thin films. $J$ Appl Phys 86, 774-778 (1999).
33. Wuttig M, Bhaskaran $\mathrm{H}$, Taubner T. Phase-change materials for non-volatile photonic applications. Nat Photonics 11, 465-476 (2017).

34. Cao T, Zhang L, Simpson RE, Cryan MJ. Mid-infrared tunable polarization-independent perfect absorber using a phasechange metamaterial. J Opt Soc Am B 30, 1580-1585 (2013).

35. Cao T, Simpson RE, Cryan MJ. Study of tunable negative index metamaterials based on phase-change materials. J Opt Soc Am B 30, 439-444 (2013).

36. Kwon Y, Kim JH, Chae S, Lee Y, Jachun SG et al. Device characteristics of a Ge-doped $\mathrm{SbTe}$ alloy for high-speed phasechange random access memory. J Korean Phys Soc 59, 466-469 (2011).

37. Wuttig M. Towards a universal memory. Nat Mater 4, 265-266 (2005).

38. Gholipour B, Zhang JF, MacDonald KF, Hewak DW, Zheludev NI. An all-optical, non-volatile, bidirectional, phase-change meta-switch. Adv Mater 25, 3050-3054 (2013).

39. Michel AKU, Zalden $P$, Chigrin DN, Wuttig M, Lindenberg AM et al. Reversible optical switching of infrared antenna resonances with ultrathin phase-change layers using femtosecond laser pulses. ACS Photonics 1, 833-839 (2014).

40. Cao T, Fang LH, Cao Y, Li N, Fan ZY et al. Dynamically reconfigurable topological edge state in phase change photonic crystals. Sci Bull 64, 814-822 (2019).

41. Kalikka J, Zhou XL, Behera J, Nannicini G, Simpson RE. Evolutionary design of interfacial phase change van der Waals heterostructures. Nanoscale 8, 18212-18220 (2016).

42. Zhou XL, Behera JK, Lv SL, Wu LC, Song ZT et al. Avalanche atomic switching in strain engineered $\mathrm{Sb}_{2} \mathrm{Te}_{3}-\mathrm{GeTe}$ interfacial phase-change memory cells. Nano Futures 1, 025003 (2017).

43. Chu CH, Tseng ML, Chen J, Wu PC, Chen YH et al. Active dielectric metasurface based on phase-change medium. Laser Photon Rev 10, 986-994 (2016).

44. Zhou XL, Kalikka J, Ji XL, Wu LC, Song ZT et al. Phase-change memory materials by design: a strain engineering approach. Adv Mater 28, 3007-3016 (2016).

45. Behera JK, Zhou XL, Tominaga J, Simpson RE. Laser switching and characterisation of chalcogenides: systems, measurements, and applicability to photonics [invited]. Opt Mater Express 7, 3741-3759 (2017).

46. Kodama $\mathrm{CH}$, Coutu Jr RA. Tunable split-ring resonators using germanium telluride. Appl Phys Lett 108, 231901 (2016).

47. Pitchappa P, Kumar A, Prakash S, Jani H, Venkatesan T et al. Chalcogenide phase change material for active terahertz photonics. Adv Mater 31, 1808157 (2019).

48. Chew LT, Dong WL, Liu L, Zhou XL, Behera J et al. Chalcogenide active photonics. Proc SPIE 10345, 103451B (2017).

49. Azad AK, Zhang WL. Resonant terahertz transmission in subwavelength metallic hole arrays of sub-skin-depth thickness. Opt Lett 30, 2945-2947 (2005).

50. Gopalan P, Wang YS, Sensale-Rodriguez B. Terahertz characterization of two-dimensional low-conductive layers enabled by metal gratings. Sci Rep 11, 2833 (2021).

51. Pendry JB, Martín-Moreno L, Garcia-Vidal FJ. Mimicking surface plasmons with structured surfaces. Science $\mathbf{3 0 5}, 847-848$ (2004).

52. Lalanne P, Rodier JC, Hugonin JP. Surface plasmons of metallic surfaces perforated by nanohole arrays. J Opt A:Pure Appl Opt 7, 422-426 (2005). 
53. Liu HT, Lalanne P. Microscopic theory of the extraordinary optical transmission. Nature 452, 728-731 (2008).

54. Liu HT, Lalanne P. Comprehensive microscopic model of the extraordinary optical transmission. J Opt Soc Am A 27, 2542-2550 (2010).

55. van Beijnum F, Rétif $C$, Smiet CB, Liu HT, Lalanne $P$ et al. Quasi-cylindrical wave contribution in experiments on extraordinary optical transmission. Nature 492, 411-414 (2012).

56. Ebbesen TW, Lezec HJ, Ghaemi HF, Thio T, Wolff PA. Extraordinary optical transmission through sub-wavelength hole arrays. Nature 391, 667-669 (1998).

57. Martín-Moreno L, García-Vidal F, Lezec HJ, Pellerin KM, Thio T et al. Theory of extraordinary optical transmission through subwavelength hole arrays. Phys Rev Lett 86, 1114 (2001).

58. Porto JA, García-Vidal F, Pendry JB. Transmission resonances on metallic gratings with very narrow slits. Phys Rev Lett $\mathbf{8 3}$, 2845-2848 (1999).

59. Lee BS, Burr GW, Shelby RM, Raoux S, Rettner CT et al. Observation of the role of subcritical nuclei in crystallization of a glassy solid. Science 326, 980-984 (2009).

60. Wang L, Zhang $Y X$, Guo XQ, Chen $T$, Liang $H J$ et al. A review of $\mathrm{THz}$ modulators with dynamic tunable metasurfaces. Nanomaterials 9, 965 (2019).

61. Ma ZT, Geng ZX, Fan ZY, Liu J, Chen HD. Modulators for terahertz communication: the current state of the art. Research 2019, 6482975 (2019).

62. Raoux S. Phase change materials. Annu Rev Mater Res 39, 25-48 (2009).

63. Terao M, Morikawa T, Ohta T. Electrical phase-change memory: fundamentals and state of the art. Jpn J Appl Phys 48, 080001 (2009).

64. Orava J, Greer AL, Gholipour B, Hewak DW, Smith CE. Characterization of supercooled liquid $\mathrm{Ge}_{2} \mathrm{Sb}_{2} \mathrm{Te}_{5}$ and its crystallization by ultrafast-heating calorimetry. Nat Mater 11, 279-283 (2012).

65. Chu CH, Da Shiue C, Cheng HW, Tseng ML, Chiang HP et al. Laser-induced phase transitions of $\mathrm{Ge}_{2} \mathrm{Sb}_{2} \mathrm{Te}_{5}$ thin films used in optical and electronic data storage and in thermal lithography. Opt Express 18, 18383-18393 (2010).

66. Yamada N, Ohno E, Nishiuchi K, Akahira N, Takao M. Rapidphase transitions of $\mathrm{GeTe}-\mathrm{Sb}_{2} \mathrm{Te}_{3}$ pseudobinary amorphous thin films for an optical disk memory. J Appl Phys 69, 2849-2856 (1991).

67. Weidenhof V, Pirch N, Friedrich I, Ziegler S, Wuttig M. Minimum time for laser induced amorphization of $\mathrm{Ge}_{2} \mathrm{Sb}_{2} \mathrm{Te}_{5}$ films. $J$ Appl Phys 88, 657-664 (2000).

68. Wuttig M, Salinga M. Fast transformers. Nat Mater 11, 270-271 (2012).

69. Oh SH, Baek K, Son SK, Song K, Oh JW et al. In situ TEM observation of void formation and migration in phase change memory devices with confined nanoscale $\mathrm{Ge}_{2} \mathrm{Sb}_{2} \mathrm{Te}_{5}$. Nanoscale Adv 2, 3841-3848 (2020).

70. Sarkar J, Gleixner B. Evolution of phase change memory characteristics with operating cycles: electrical characterization and physical modeling. Appl Phys Lett 91, 233506 (2007).

71. Do K, Lee $D, K o D H$, Sohn $H$, Cho MH. TEM study on volume changes and void formation in $\mathrm{Ge}_{2} \mathrm{Sb}_{2} \mathrm{Te}_{5}$ films, with repeated phase changes. Electrochem Solid State Lett 13, H284-H286 (2010).

72. Kim C, Kang DM, Lee TY, Kim KHP, Kang YS et al. Direct evidence of phase separation in $\mathrm{Ge}_{2} \mathrm{Sb}_{2} \mathrm{Te}_{5}$ in phase change memory devices. Appl Phys Lett 94, 193504 (2009).

73. Krusin-Elbaum L, Cabral Jr C, Chen KN, Copel M, Abraham DW et al. Evidence for segregation of $\mathrm{Te}$ in $\mathrm{Ge}_{2} \mathrm{Sb}_{2} \mathrm{Te}_{5}$ films: effect on the "phase-change" stress. Appl Phys Lett 90, 141902 (2007).

74. Yang TY, Park IM, Kim BJ, Joo YC. Atomic migration in molten and crystalline $\mathrm{Ge}_{2} \mathrm{Sb}_{2} \mathrm{Te}_{5}$ under high electric field. Appl Phys Lett 95, 032104 (2009).

75. Haynes WM, Lide DR, Bruno TJ. CRC Handbook of Chemistry and Physics 95th ed (CRC Press, Boca Raton, 2014).

76. Chen HT, O'hara JF, Azad AK, Taylor AJ, Averitt RD et al. Experimental demonstration of frequency-agile terahertz metamaterials. Nat Photonics 2, 295-298 (2008).

77. Yang K, Liu SC, Arezoomandan S, Nahata A, Sensale-Rodriguez B. Graphene-based tunable metamaterial terahertz filters. Appl Phys Lett 105, 093105 (2014).

78. Jung $\mathrm{H}, \mathrm{Koo} \mathrm{J}, \mathrm{Heo} \mathrm{E}, \mathrm{Cho} \mathrm{B}, \mathrm{In} \mathrm{C}$ et al. Electrically controllable molecularization of terahertz meta-atoms. Adv Mater $\mathbf{3 0}$, 1802760 (2018).

79. Deng ZL, Zhang S, Wang GP. A facile grating approach towards broadband, wide-angle and high-efficiency holographic metasurfaces. Nanoscale 8, 1588-1594 (2016).

80. Deng ZL, Cao YY, Li XP, Wang GP. Multifunctional metasurface: from extraordinary optical transmission to extraordinary optical diffraction in a single structure. Photonics Res $\mathbf{6}$, 443-450 (2018).

\section{Acknowledgements}

Tun Cao acknowledges support from the National Key Research and Development Program of China (2020YFA0714504, 2019YFA0709100) and LiaoNing Revitalization Talents Program (Grant No. XLYC1807237).

\section{Competing interests}

The authors declare no competing financial interests.

\section{Supplementary information}

Supplementary information for this paper is available at https://doi.org/10.29026/oes.2022.210010 